\title{
A generalized statistical complexity measure: Applications to quantum systems
}

\author{
R. López-Ruiz, ${ }^{1, a)}$ Á. Nagy, ${ }^{2}$ E. Romera, ${ }^{3}$ and J. Sañudo ${ }^{4}$ \\ ${ }^{1}$ DIIS and BIFI, Facultad de Ciencias, Universidad de Zaragoza, E-50009 Zaragoza, \\ Spain \\ ${ }^{2}$ Department of Theoretical Physics, University of Debrecen, H-4010 Debrecen, Hungary \\ ${ }^{3}$ Departamento de Física Atómica, Molecular y Nuclear and Instituto Carlos I de \\ Física Teórica y Computacional, Universidad de Granada, E-18071 Granada, Spain \\ ${ }^{4}$ Departamento de Física, Facultad de Ciencias, Universidad de Extremadura, E-06071 \\ Badajoz, Spain, and BIFI, Universidad de Zaragoza, E-50009 Zaragoza, Spain
}

(Received 20 May 2009; accepted 19 November 2009; published online 31 December 2009)

\begin{abstract}
A two-parameter family of complexity measures $\widetilde{C}^{(\alpha, \beta)}$ based on the Rényi entropies is introduced and characterized by a detailed study of its mathematical properties. This family is the generalization of a continuous version of the Lopez-RuizMancini-Calbet complexity, which is recovered for $\alpha=1$ and $\beta=2$. These complexity measures are obtained by multiplying two quantities bringing global information on the probability distribution defining the system. When one of the parameters, $\alpha$ or $\beta$, goes to infinity, one of the global factors becomes a local factor. For this special case, the complexity is calculated on different quantum systems: H-atom, harmonic oscillator, and square well. (C) 2009 American Institute of Physics. [doi:10.1063/1.3274387]
\end{abstract}

\section{INTRODUCTION}

The question concerning the quantification of complexity ${ }^{1}$ has been addressed in many different fields, from computer science to physics. Depending on the properties to be grasped different answers are found in the literature. ${ }^{2-5}$ The study of these statistical measures in physical systems, and, in particular, in quantum systems, has a role of growing importance. So, information entropies and statistical complexities have been calculated on different atomic systems. ${ }^{6,7}$ In particular, the so-called Lopez-Ruiz-Mancini-Calbet (LMC) complexity ${ }^{8-10}$ has been computed in the position and momentum spaces for the density functions of the hydrogenlike atoms and the quantum isotropic harmonic oscillator. ${ }^{11,12}$ It has been found that the minimum values of that statistical measure is taken on the quantum states with the highest orbital angular momentum, just those wave functions that correspond to the Bohr-like orbits in the prequantum image.

Many LMC-like statistical complexities are defined as a product of two factors, one of them measuring the broadening of the distribution that defines the system and the other one quantifying the narrowness of it. Both factors are global magnitudes that can be calculated by integrating over the whole support of the distribution.

Shannon information ${ }^{13}$ is an adequate indicator to grasp the spreading of a distribution and thus it is employed as a basic ingredient of the first factor of complexity measures. Concretely, it plays an important role in the original LMC statistical complexity in which the second factor, the so-called disequilibrium, ${ }^{8}$ was originally chosen to be the square distance to the equiprobability distribution. Other functions can be used to define different families of LMC-like complexities. ${ }^{14}$ When the simple power Shannon entropy is taken as the first factor, it is a meaningful parameter to characterize the shape of a distribution. ${ }^{9}$ In this particular case, the log of the LMC complexity coincides with the structural entropy introduced to study questions concerning quantum

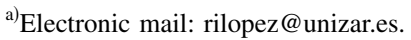


localization. ${ }^{15,16}$ Other generalizations of the Shannon information that depend on a parameter ${ }^{14,17,18}$ can also be used. For instance, the Rényi entropy, that is, related with relevant physical magnitudes in atomic physics, ${ }^{19,20}$ is a good candidate to be adopted in this purpose.

Hence, in this work, we undertake the generalization of the second factor of the LMC complexity by means of the Rényi entropy. We apply the same procedure in Ref. 21 where the first factor was generalized. This is presented in Sec. II. Some properties of this new two-parameterdependent complexity measure are indicated in Sec. III. Strikingly, when one of the parameters tends to infinity, the asymptotic limit of this measure becomes the product of a global quantity by a local one. In Sec. IV, the analysis and calculation of this special global/local product case of the generalized complexity measure are performed for the H-atom, the quantum harmonic oscillator, and the square well. Section $\mathrm{V}$ includes the conclusions.

\section{GENERALIZED STATISTICAL COMPLEXITY MEASURE $\tilde{\boldsymbol{C}}_{f}^{(\alpha, \beta)}$}

Let us consider a $D$-dimensional density function $f(\mathbf{r})$ [with $f(\mathbf{r})$ nonnegative and $\int f(\mathbf{r}) d \mathbf{r}$ $=1$ ]. The Rényi entropy of order $\alpha$ of the density function $f$ is given by

$$
R_{f}^{(\alpha)}=\frac{1}{1-\alpha} \ln \int[f(\mathbf{r})]^{\alpha} d \mathbf{r} \quad \text { for } 0<\alpha<\infty, \quad \alpha \neq 1,
$$

where $\mathbf{r}$ stands for $r_{1}, \ldots, r_{D}$. From the above definition, it is straightforward to see that in the limit $\alpha \rightarrow 1$ we have $R_{f}^{(\alpha)} \rightarrow S_{f}=-\int f(\mathbf{r}) \ln f(\mathbf{r}) d \mathbf{r}$, with $S_{f}$ the Shannon entropy of $f$, and in the limit $\alpha$ $\rightarrow \infty$ then $R_{f}^{(\alpha)} \rightarrow-\ln \|f\|_{\infty}$, where $\|f\|_{\infty}=\sup _{\mathbf{r}} f(\mathbf{r})$ represents the maximum reached by $f$ over its whole support.

The importance of Rényi entropies comes from the fact that, for atoms and molecules, density functionals (kinetic energy, exchange energy, and classical Coulomb repulsion energy) can be expanded in terms of the local homogeneous functionals $\exp (1-\alpha) R_{\rho}^{(\alpha)} \cdot{ }^{19,20}$ In particular, it is well known that for $\alpha=5 / 3, \alpha=4 / 3$, and $\alpha=2$, the local density approximations are related to the kinetic and exchange energies and the average of the density, respectively. Also the case when $\alpha$ goes to infinity has physical significance: it contains information about the maximum of the probability density, which in the ground state of atoms is taken at the nucleus. In this situation, let us recall the importance of the density in the cusp due to Kato's theorem.

A continuous version ${ }^{9}$ of the measure of complexity $C_{f}$, the so-called LMC complexity introduced in Ref. 8, is defined by

$$
C_{f}=H_{f} Q_{f}, \quad \text { with } H_{f}=e^{S_{f}} \text { and } Q_{f}=e^{-R_{f}^{(2)}} .
$$

When the Shannon entropy of the statistical complexity $C_{f}$ is replaced with the Rényi entropy of order $\alpha$, we obtain the generalized statistical measure of complexity $C_{f}^{(\alpha)}$, which has been defined by $^{21}$

$$
C_{f}^{(\alpha)}=H_{f}^{(\alpha)} Q_{f}, \quad \text { with } H_{f}^{(\alpha)}=e^{R_{f}^{(\alpha)}},
$$

and tends to $C_{f}$ in the limit $\alpha \rightarrow 1$.

Now we can substitute in a symmetric way the $R_{f}^{(2)}$ ingredient of the above complexities for the Rényi entropy of order $\beta$, which allows us to obtain a $(\alpha, \beta)$-dependent measure of complexity $\widetilde{C}_{f}^{(\alpha, \beta)}$, which is defined by

$$
\widetilde{C}_{f}^{(\alpha, \beta)}=e^{R_{f}^{(\alpha)}-R_{f}^{(\beta)}}, \quad 0<\alpha, \quad \beta<\infty .
$$

So, we recover $\widetilde{C}_{f}^{(1,2)}=C_{f}$ and $\widetilde{C}_{f}^{(\alpha, 2)}=C_{f}^{(\alpha)}$ of Refs. 8 and 21 , respectively. This type of generalization based on Rényi entropies differences was suggested in Refs. 22 and 23. Taking into account that $\|f\|_{p}=\left(\int f^{p}\right)^{1 / p}, \widetilde{C}^{(\alpha, \beta)}$ can also be expressed as 


$$
\widetilde{C}_{f}^{(\alpha, \beta)}=\|f\|_{\alpha}^{\alpha /(1-\alpha)} \cdot\|f\|_{\beta}^{\beta /(\beta-1)}
$$

Here, we suppose that the integral of the $p$ th power of the probability density exists. It might happen, however, that for certain power(s) the integral does (do) not exist. Even if there are values of $\alpha$ for which Eq. (5) diverge, we can find several other values of $\alpha$ for which the new complexity measure takes a finite value. In this sense, the generalized complexity extends the complexity measure to any kind of well behaved distribution.

\section{PROPERTIES OF $\tilde{\boldsymbol{C}}_{f}^{(\alpha, \beta)}$}

Now, having as guideline Ref. 9, we proceed to present some mathematical properties of this new generalized statistical complexity measure.

\section{A. Inversion symmetry}

It is straightforward to check that

$$
\widetilde{C}_{f}^{(\alpha, \beta)} \widetilde{C}_{f}^{(\beta, \alpha)}=1
$$

and then $\widetilde{C}_{f}^{(\alpha, \alpha)}=1$.

\section{B. Monotonicity and universal bound}

Taking into account that the Rényi entropy is a nonincreasing function of $\alpha$, it can be easily proven the following.

(i) $\quad \widetilde{C}_{f}^{(\alpha, \beta)} \geq 1$ if $\alpha<\beta$ and $\widetilde{C}_{f}^{(\alpha, \beta)} \leq 1$ if $\alpha>\beta$.

(ii) $\widetilde{C}_{f}^{(\alpha, \beta)}$ is a nonincreasing function of $\alpha$ for a fixed $\beta$ and an increasing function of $\beta$ for a fixed $\alpha$.

(iii) The lower (upper) bound 1 is reached for $\alpha<\beta(\alpha>\beta)$ for the rectangular density function. It is a universal bound independent of $\alpha$ and $\beta$, as it is shown in Sec. III F.

\section{Invariance under translations and rescaling transformations}

$\widetilde{C}_{f}^{(\alpha, \beta)}$ is invariant under scaling transformations, i.e., for $f_{\lambda}=\lambda^{D} f(\lambda \mathbf{r})$, then $\widetilde{C}_{f_{\lambda}}^{(\alpha, \beta)}=\widetilde{C}_{f}^{(\alpha, \beta)}$. Also, it is invariant under translations. Hence, in general, let us consider a scaling transformation and a translation parametrized by $(a, \mathbf{b})$, respectively, of the form

$$
f_{a \mathbf{b}}(\mathbf{r})=a^{D} f[a(\mathbf{r}-\mathbf{b})],
$$

where the distribution function $f$ is normalized, $\int f(\mathbf{r}) d \mathbf{r}=1$.

The Rényi entropy of order $\alpha$ is transforming as

$$
R_{a \mathbf{b}}^{(\alpha)}=\frac{1}{1-\alpha} \ln \int\left(a^{D} f[a(\mathbf{r}-\mathbf{b})]\right)^{\alpha} d \mathbf{r}=R^{(\alpha)}-D \ln a,
$$

where a change in variable $\mathbf{y}=a(\mathbf{r}-\mathbf{b})$ was applied. Note that there is no dependence on the parameter $\mathbf{b}$. Therefore, the new complexity measure $\widetilde{C}_{f}^{(\alpha, \beta)}(4)$ is invariant under this transformation.

\section{Invariance under replication}

Different types of replication can be defined. Here we show the invariance of the complexity measure for one particular type of replication. Take $n$ copies $f_{m}(\mathbf{r}), m=1, \ldots, n$ of the distribution function $f(\mathbf{r})$, 


$$
f_{m}(\mathbf{r})=n^{D / 2-1} f\left[n^{1 / 2}\left(\mathbf{r}-\mathbf{b}_{m}\right)\right], \quad 1 \leq m \leq n,
$$

where the support of each $f_{m}(\mathbf{r})$ is centered at the point $\mathbf{b}_{m}$ and the supports are disjoint. We can immediately obtain that $\int f_{m}(\mathbf{r}) d \mathbf{r}=1 / n$. Then we can define the distribution function, ${ }^{9}$

$$
q(\mathbf{r})=\sum_{m=1}^{n} f_{m}(\mathbf{r}),
$$

that is normalized, $\int q(\mathbf{r}) d \mathbf{r}=1$. From Eq. (9) we can easily calculate that

$$
\int f_{m}^{\alpha}(\mathbf{r}) d \mathbf{r}=n^{(\alpha-1) D / 2-\alpha} \int f^{\alpha}(\mathbf{y}) d \mathbf{y} .
$$

As the replicas are supported on disjoint sets, we have

$$
\sum_{m=1}^{n} \int f_{m}^{\alpha}(\mathbf{r}) d \mathbf{r}=n^{(\alpha-1)(D / 2-1)} \int f^{\alpha}(\mathbf{y}) d \mathbf{y} .
$$

Then the transformation of the Rényi entropy of order $\alpha$ is

$$
R_{q}^{(\alpha)}=R_{f}^{(\alpha)}-(D / 2-1) \ln n .
$$

Hence the complexity measure $\widetilde{C}_{q}^{(\alpha, \beta)}(4)$ is replica invariant.

\section{E. Near continuity}

Take two distribution functions $f(\mathbf{r})$ and $g(\mathbf{r})$ defined on the set $M$ in the $D$-dimensional space considered. Let $\delta$ be a positive real number. The functions $f(\mathbf{r})$ and $g(\mathbf{r})$ are $\delta$-neighboring functions on $M$, if the Lebesgue measure of the points $\mathbf{r} \in M$ satisfying $|f(\mathbf{r})-g(\mathbf{r})| \geq \delta$ is zero. A functional $T$ of the distribution functions is near continuous if for any $\varepsilon>0$ there exists $\delta(\varepsilon)$ $>0$, such that for any $\delta$-neighboring functions $f(\mathbf{r})$ and $g(\mathbf{r})$ on $T$, then $|T(f)-T(g)|<\varepsilon$.

Take the function

$$
g_{\delta, B}(\mathbf{r})= \begin{cases}\frac{1-\delta}{c_{D}} & \text { if }|\mathbf{r}|<1 \\ \frac{\delta}{c_{D}\left(B^{D}-1\right)} & \text { if } 1<|\mathbf{r}|<B \\ 0 & \text { otherwise, }\end{cases}
$$

where

$$
c_{D}=\frac{2 \pi^{D / 2}}{D \Gamma(D / 2)},
$$

where $B>1$ and $1>\delta>0$. As $c_{D}$ is the volume of a unity $D$-dimensional sphere then $g$ is normalized to 1 . One can easily calculate the Rényi entropy of order $\alpha$,

$$
R_{g}^{(\alpha)}=\frac{1}{1-\alpha} \ln \left[(1-\delta)^{\alpha}+\frac{\delta^{\alpha}}{\left(B^{D}-1\right)^{\alpha-1}}\right]+\ln c_{D} .
$$

From here, we obtain $\widetilde{C}_{g}^{(\alpha, \beta)}=e^{R_{g}^{(\alpha)}-R_{g}^{(\beta)}}, 0<\alpha, \beta<\infty$. Consider now the rectangular density function, 


$$
\chi(\mathbf{r})= \begin{cases}\frac{1}{c_{D}} & \text { if }|\mathbf{r}|<1 \\ 0 & \text { otherwise }\end{cases}
$$

The Rényi entropy of order $\alpha$,

$$
R_{\chi}^{(\alpha)}=\ln c_{D},
$$

does not depend on the $\alpha$. Consequently, we are led to the result

$$
\widetilde{C}_{\chi}^{(\alpha, \beta)}=1 .
$$

Note that $g_{\delta, B}$ and $\chi$ are $\widetilde{\delta}$-neighboring functions for $0<\delta<\widetilde{\delta}<1$ and

$$
\lim _{\delta \rightarrow 0} R_{g}^{(\alpha)}=R_{\chi}^{(\alpha)}=\ln c_{D} .
$$

Therefore, in the limit $\delta \rightarrow 0$ the complexity measure $\widetilde{C}^{(\alpha, \beta)}$ takes the same value,

$$
\lim _{\delta \rightarrow 0} \widetilde{C}_{g}^{(\alpha, \beta)}=\widetilde{C}_{\chi}^{(\alpha, \beta)}=1 .
$$

The importance of a bounded support to obtain this result deserves some longer explanation to be done in a future work, such as it was suggested in Ref. 9.

\section{F. The extremal complexity}

Naturally, the extremal complexity, as it happens with the structural entropy, ${ }^{15}$ is reached for the rectangular function [Eqs. (17) and (19)]. Here, by following Ref. 9, we prove that this is only distribution reaching the minimal complexity. The function $f$ is taken as a sum of rectangular functions $\chi_{E_{k}}$ defined on disjoint sets $E_{k}, k=1, \ldots, n$ with Lebesgue measure $\mu_{k}$,

$$
f(\mathbf{r})=\sum_{k=1}^{n} \lambda_{k} \chi_{E_{k}}
$$

Its integrals can be easily calculated,

$$
\int f^{\alpha} d \mathbf{r}=\sum_{k=1}^{n} \lambda_{k}^{\alpha} \mu_{k}
$$

The logarithm of complexity measure $\widetilde{C}^{(\alpha, \beta)}$ has the form

$$
\ln \widetilde{C}^{(\alpha, \beta)}=\frac{1}{1-\alpha} \ln \left(\sum_{k=1}^{n} \lambda_{k}^{\alpha} \mu_{k}\right)-\frac{1}{1-\beta} \ln \left(\sum_{k=1}^{n} \lambda_{k}^{\beta} \mu_{k}\right) .
$$

We seek the extremum of the logarithm of complexity measure $\widetilde{C}^{(\alpha, \beta)}$ under the normalization condition,

$$
\sum_{k=1}^{n} \lambda_{k} \mu_{k}=1
$$

The variation with respect to $\lambda_{k}$ and $\mu_{k}$ after straightforward manipulation leads the equations 


$$
\lambda_{k}^{\alpha-\beta}=\frac{\sum_{l=1}^{n} \lambda_{l}^{\alpha} \mu_{l}}{\sum_{l=1}^{n} \lambda_{l}^{\beta} \mu_{l}}
$$

for all $k=1, \ldots, n$. As $\lambda_{k}$ has the same value for all $k$, then $f$ is a rectangular function. Moreover, with the help of the near-continuity property as explained in Ref. 9, it can also be argued that the rectangular distribution is the only distribution reaching the extremal complexity.

\section{G. The cases $\tilde{C}_{f}^{(\alpha, \infty)}$ and $\tilde{C}_{f}^{(\alpha, 0)}$}

As before explained, the new complexity measure has a completely different behavior for $\alpha$ $<\beta$ and $\alpha>\beta$. In the first case, there is a lower bound, and in the second case there is an upper bound. Both are universal, i.e., the bound is equal to 1 for any (finite and nonzero) value of $\alpha$ or $\beta$, and this universal bound is reached at the rectangular density function. This is a consequence of the fact that the Rényi entropy is independent of the parameter $\alpha$ or $\beta$, then the new complexity measure has the value of 1 for any $\alpha$ or $\beta$.

Finally, let us remark that when $\beta$ goes to infinity a special case of the complexity measure in terms of a local quantity $\left(\|f\|_{\infty}=\sup _{\mathbf{r}} f(\mathbf{r})\right)$ is obtained: $\widetilde{C}_{f}^{(\alpha, \infty)}=e^{R_{f}^{(\alpha)}}\|f\|_{\infty}$. To prove this property it is sufficient to take into account that $\lim _{p \rightarrow \infty}\left(\int f(\mathbf{r})^{p} d \mathbf{r}\right)^{1 / p}=\sup _{\mathbf{r}} f(\mathbf{r}) .^{24}$ This complexity measure verifies $\widetilde{C}_{f}^{(\alpha, \infty)}>1$ for all $\alpha$ (and $\widetilde{C}_{f}^{(\infty, \alpha)}<1$ ). We can also mention that $\widetilde{C}_{f}^{(\alpha, 0)} \rightarrow 0$ for finite $\alpha$ and $\beta \rightarrow 0$.

\section{CALCULATION OF $\tilde{\boldsymbol{C}}_{\boldsymbol{f}}^{(\alpha, \infty)}$ ON DIFFERENT QUANTUM SYSTEMS}

Among the different statistical indicators that have been defined as a product of two entropic terms, the generalized complexity $\widetilde{C}^{(\alpha, \beta)}$ is also obtained by multiplying two factors, each one of them bringing global information on the distribution $f$. Recall, however, that the limit $\widetilde{C}_{f}^{(\alpha, \infty)}$ combines global information on the distribution $f$, just the part corresponding to $e^{R_{f}^{(\alpha)}}$, and local information coming from only a specific point of the space where the distribution $f$ is supported, indeed the maximum of the density, $\|f\|_{\infty}$. Equivalently, the limit version $\widetilde{C}_{f}^{(\infty, \beta)}$ presents a symmetrical behavior with respect to $\widetilde{C}_{f}^{(\alpha, \infty)}$ by considering the property $\widetilde{C}_{f}^{(\infty, \sigma)} \widetilde{C}_{f}^{(\sigma, \infty)}=1$. Here we undertake the calculation of $\widetilde{C}_{f}^{(\alpha, \infty)}$ for three paradigmatic quantum systems: the $H$-atom, the harmonic oscillator, and the square well.

\section{A. $\boldsymbol{H}$-atom}

The atomic state of the $H$-atom determined by the quantum numbers $(n, l, m)$ in position space $[\vec{r}=(r, \Omega)$, with $r$ the radial distance and $\Omega$ the solid angle] is given by the nonrelativistic wave function,

$$
\Psi_{n, l, m}(\vec{r})=R_{n, l}(r) Y_{l, m}(\Omega),
$$

where $R_{n, l}(r)$ is the radial part and $Y_{l, m}(\Omega)$ is the spherical harmonic. The radial part is expressed as $^{25}$

$$
R_{n, l}(r)=\frac{2}{n^{2}}\left[\frac{(n-l-1) !}{(n+l) !}\right]^{1 / 2}\left(\frac{2 r}{n}\right)^{l} e^{-r / n} L_{n-l-1}^{2 l+1}\left(\frac{2 r}{n}\right),
$$

$L_{\alpha}^{\beta}(t)$ being the associated Laguerre polynomials. Atomic units are used in this section.

The same hydrogenic atomic state in momentum space $[\vec{p}=(p, \hat{\Omega})$, with the momentum modulus $p$ and the solid angle $\hat{\Omega}]$ is given by the wave function 

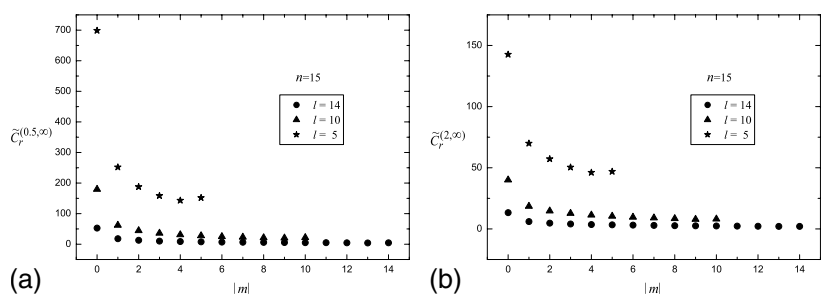

FIG. 1. Generalized statistical complexity in position space, $\widetilde{C}_{r}^{(\alpha, \infty)}$, vs $|m|$ for different $l$ values when $n=15$ in the hydrogen atom. (a) $\alpha=0.5$ and (b) $\alpha=2$. All values are in atomic units.

$$
\hat{\Psi}_{n, l, m}(\vec{p})=\hat{R}_{n, l}(p) Y_{l, m}(\hat{\Omega}),
$$

where the radial part $\hat{R}_{n, l}(p)$ is expressed now as ${ }^{26}$

$$
\hat{R}_{n, l}(p)=\left[\frac{2}{\pi} \frac{(n-l-1) !}{(n+l) !}\right]^{1 / 2} n^{2} 2^{2 l+2} l ! \frac{n^{l} p^{l}}{\left(n^{2} p^{2}+1\right)^{l+2}} G_{n-l-1}^{l+1}\left(\frac{n^{2} p^{2}-1}{n^{2} p^{2}+1}\right)
$$

with the Gegenbauer polynomials $G_{\alpha}^{\beta}(t)$.

Taking the former expressions, the probability density in position and momentum spaces,

$$
\rho(\vec{r})=\left|\Psi_{n, l, m}(\vec{r})\right|^{2}, \quad \gamma(\vec{p})=\left|\hat{\Psi}_{n, l, m}(\vec{p})\right|^{2},
$$

can be explicitly calculated. From these densities, we compute $\widetilde{C}_{f}^{(\alpha, \infty)}$, taking into account that for these cases $f=\rho(\vec{r})$ or $f=\gamma(\vec{p})$, respectively.

In Fig. 1, the value of the generalized complexity in position space, $\widetilde{C}_{r}^{(\alpha, \infty)}$, is shown for $n$ $=15$ and $l=5,10,14$ versus $|m|$ with $\alpha=0.5$ [Fig. 1(a)] and $\alpha=2$ [Fig. 1(b)]. The same calculation in momentum space, $\widetilde{C}_{p}^{(\alpha, \infty)}$, can be seen in Figs. 2(a) and 2(b) for the cases $\alpha=0.5$ and $\alpha=2$, respectively. Note that the minimum of the generalized complexity corresponds just to the orbitals with the highest $l$ for a given $n$ in both position and momentum spaces, that for a large $n$ converge to the Bohr orbits in the prequantum image. Moreover, due to the property $3 \cdot B(i)$, the behavior displayed in Figs. 1 and 2 for $\alpha=0.5$ and $\alpha=2$ is qualitatively independent of the parameter $\alpha$.

\section{B. Harmonic oscillator}

Let us consider a particle under the action of the potential energy $V(r)=\lambda^{2} r^{2} / 2$, where $\lambda$ is a positive real constant expressing the potential strength. The three-dimensional nonrelativistic wave functions of this system in position space $(\vec{r}=(r, \Omega))$ are
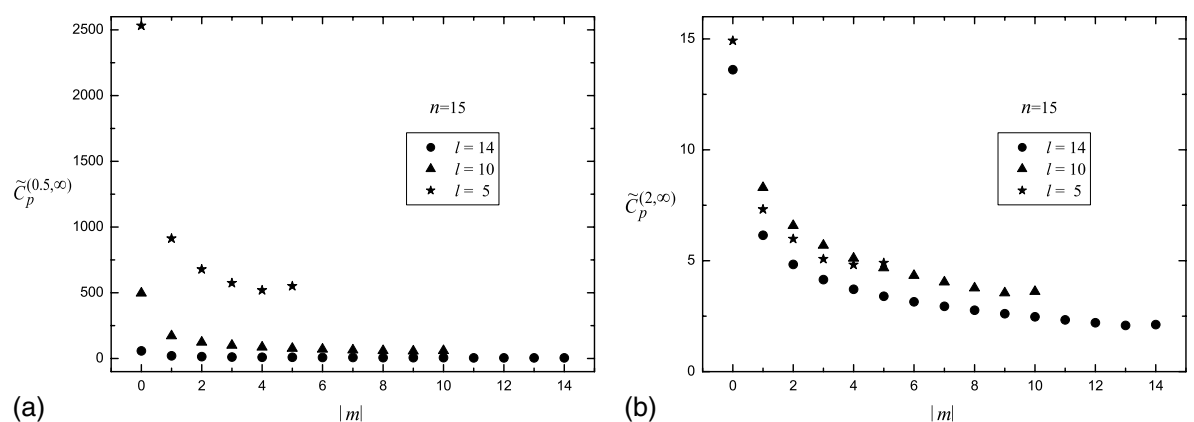

FIG. 2. Generalized statistical complexity in momentum space, $\widetilde{C}_{p}^{(\alpha, \infty)}$, vs $|m|$ for different $l$ values when $n=15$ in the hydrogen atom. (a) $\alpha=0.5$ and $\alpha=2$. All values are in atomic units. 

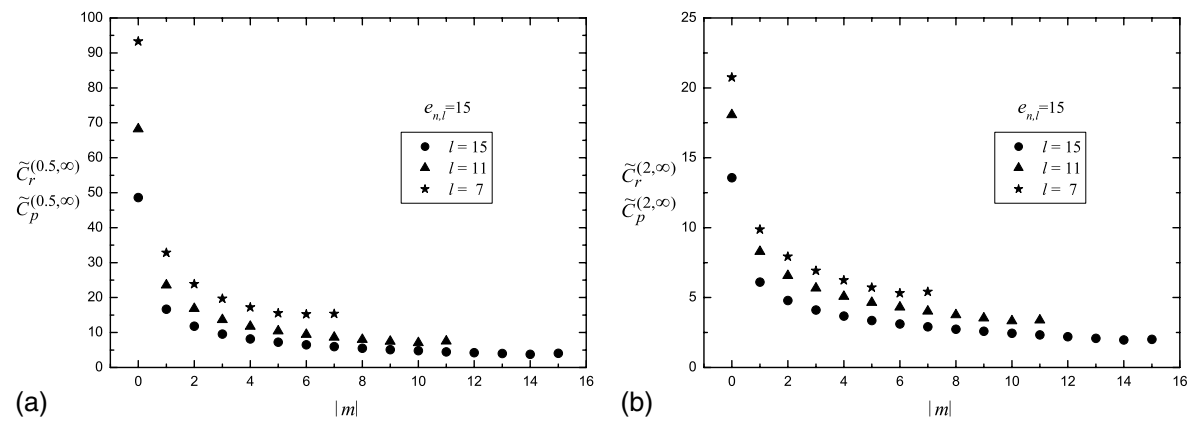

FIG. 3. Generalized statistical complexity in position space, $\widetilde{C}_{r}^{(\alpha, \infty)}$, and momentum space, $\widetilde{C}_{p}^{(\alpha, \infty)}$, vs $|m|$ for the energy $e_{n, l}=15$ in the quantum isotropic harmonic oscillator for (a) $\alpha=0.5$ and (b) $\alpha=2$. Recall that $\widetilde{C}_{r}^{(\alpha, \infty)}=\widetilde{C}_{p}^{(\alpha, \infty)}$. All values are in atomic units.

$$
\Psi_{n, l, m}(\vec{r})=R_{n, l}(r) Y_{l, m}(\Omega),
$$

where $R_{n, l}(r)$ is the radial part and $Y_{l, m}(\Omega)$ is the spherical harmonic of the quantum state determined by the quantum numbers $(n, l, m)$. The radial part is expressed as ${ }^{25}$

$$
R_{n, l}(r)=\left[\frac{2 n ! \lambda^{l+3 / 2}}{\Gamma(n+l+3 / 2)}\right]^{1 / 2} r^{l} e^{-(\lambda / 2) r^{2}} L_{n}^{l+1 / 2}\left(\lambda r^{2}\right),
$$

where $L_{\alpha}^{\beta}(t)$ are the associated Laguerre polynomials. The levels of energy are given by

$$
E_{n, l}=\lambda(2 n+l+3 / 2)=\lambda\left(e_{n, l}+3 / 2\right),
$$

where $n=0,1,2, \ldots$ and $l=0,1,2, \ldots$ Let us observe that $e_{n, l}=2 n+l$. Thus, different pairs of $(n, l)$ can give the same $e_{n, l}$, and then the same energy $E_{n, l}$.

The wave functions in momentum space $(\vec{p}=(p, \hat{\Omega}))$ are

$$
\hat{\Psi}_{n, l, m}(\vec{p})=\hat{R}_{n, l}(p) Y_{l, m}(\hat{\Omega}),
$$

where the radial part $\hat{R}_{n, l}(p)$ is now given by the expression

$$
\hat{R}_{n, l}(p)=\left[\frac{2 n ! \lambda^{-l-3 / 2}}{\Gamma(n+l+3 / 2)}\right]^{1 / 2} p^{l} e^{-p^{2} / 2 \lambda} L_{n}^{l+1 / 2}\left(p^{2} / \lambda\right) .
$$

Taking the former expressions, the probability density in position and momentum spaces,

$$
\rho_{\lambda}(\vec{r})=\left|\Psi_{n, l, m}(\vec{r})\right|^{2}, \quad \gamma_{\lambda}(\vec{p})=\left|\hat{\Psi}_{n, l, m}(\vec{p})\right|^{2},
$$

can be explicitly calculated. From these densities, the generalized statistical complexity is computed. The subindex $\lambda$ can be dropped because this indicator, $\widetilde{C}^{(\alpha, \beta)}$, is independent of the potential strength $\lambda$ due to its invariance under scaling transformation. As a consequence of this property, it is also found that this magnitude is the same in both position and momentum spaces, $\widetilde{C}_{r}^{(\alpha, \beta)}$ $=\widetilde{C}_{p}^{(\alpha, \beta)}$.

In Fig. 3, $\widetilde{C}_{r}^{(\alpha, \infty)}$ (or $\widetilde{C}_{p}^{(\alpha, \infty)}$ ) is plotted as function of the modulus of the third component $m$, $-l \leq m \leq l$, of the orbital angular momentum $l$ for different $l$ values with a fixed energy, $e_{n, l}=15$, when $\alpha=0.5$ in Fig. 3(a) and $\alpha=2$ in Fig. 3(b). It can be observed that $\widetilde{C}_{r}^{(\alpha, \infty)}$ splits again in different sets of discrete points. Note that the values associated with the orbitals with the highest $l$ give the minimum values of $\widetilde{C}_{r}^{(\alpha, \infty)}$ that are similar to the $H$-atom converge to the Bohr-like orbits in the prequantum image. Also, due to the property $3 \cdot B(i)$, the behavior displayed in Fig. 3 for $\alpha=0.5$ and $\alpha=2$ is qualitatively independent of the parameter $\alpha$. 


\section{Square well}

The eigenstates of the energy in the quantum infinite square well in position space for a particle in a box, that is, confined in the one-dimensional interval $[0, L]$, are given by the wave functions $^{27}$

$$
\varphi_{k}(x)=\sqrt{\frac{2}{L}} \sin \left(\frac{k \pi x}{L}\right), \quad k=1,2, \ldots .
$$

The probability density of the $k$ th excited state is

$$
\rho(x)=\left|\varphi_{k}(x)\right|^{2},
$$

that gives a maximum of 2 when $L$ is considered as the natural length unit in this problem. The other factor necessary to obtain the generalized statistical complexity, $\widetilde{C}_{r}^{(\alpha, \infty)}$, gives

$$
e^{R_{f}^{(\alpha)}}=\left[\frac{2^{\alpha}}{\pi} \int_{0}^{\pi} \sin ^{2 \alpha} t d t\right]^{1 /(1-\alpha)} .
$$

Then, $\widetilde{C}_{r}^{(\alpha, \infty)}=2 g(\alpha)$ with $g(\alpha)=e^{R_{f}^{(\alpha)}}$. We conclude that the statistical complexity is degenerated for all the energy eigenstates of the quantum infinite square well. Its value can be computed as a function of $\alpha$. It takes 2 for $\alpha=0$ and decays monotonically to 1 when $\alpha \rightarrow \infty$. In the general case of a particle in a $d$-dimensional box of width $L$ in each dimension, it can be also verified that complexity is degenerated for all its energy eigenstates with a constant value given by $\widetilde{C}_{r}^{(\alpha, \infty)}$ $=(2 g(\alpha))^{d}$.

\section{SUMMARY}

The generalized complexity measure defined here provides a family of complexity measures. We have performed a detailed mathematical characterization of its properties. As usual, these complexities have been defined by multiplying two factors, each one bringing global information on the probability distribution defining the system. The whole family is identified by two parameters, $\alpha$ and $\beta$. For the special case of $\beta$ going to infinity, it is remarkable that the new complexity measure is the product of a global quantity by a local information of the density distribution.

Also we have carried out the calculation of $\widetilde{C}_{f}^{(\alpha, \infty)}$ for different quantum systems: H-atom, harmonic oscillator, and square well. Let us observe that although the $\mathrm{H}$-atom seems to be a very simple system as there is only one electron and the wave functions are known exactly, the excited-state densities are expected to show more complex behavior. Similarly, it can be said for the harmonic oscillator case. Besides the radial dependence of the densities, the angular dependence is becoming more and more complicated (through the spherical harmonics, of course) as the degree of excitation is increased. It is remarkable that the new complexity measure shows some simple systematic tendency reflected in the nicely fallen curves of Figs. 1-3 that we are not able to explore by a direct inspection of the shape of the excited-state densities. This behavior of the complexity for this specific case of global/local term product is similar to that displayed by it in the general case of global/global term product.

\section{ACKNOWLEDGMENTS}

Á.N. acknowledges a grant from the Hungarian Scientific Research Fund (OTKA) No. K 67923. E.R. acknowledges the Spanish project Grant No. FQM-2725 (Junta de Andalucía) and to FIS2008-01143. R.L.R. and J.S. acknowledge some financial support from the Spanish project Grant No. DGICYT-FIS2009-13364-C02-01.

${ }^{1}$ S. Hawking, "I think the next century will be the century of complexity," in: San José Mercury News, 23 January 2000.

${ }^{2}$ A. N. Kolmogorov, Probl. Inf. Transm. 1, 1 (1965).

${ }^{3}$ G. Chaitin, J. ACM 13, 547 (1966). 
${ }^{4}$ A. Lempel and J. Ziv, IEEE Trans. Inf. Theory 22, 75 (1976).

${ }^{5}$ J. P. Crutchfield and K. Young, Phys. Rev. Lett. 63, 105 (1989).

${ }^{6}$ S. R. Gadre, S. B. Sears, S. J. Chakravorty, and R. D. Bendale, Phys. Rev. A 32, 2602 (1985).

${ }^{7}$ K. Ch. Chatzisavvas, Ch. C. Moustakidis, and C. P. Panos, J. Chem. Phys. 123, 174111 (2005).

${ }^{8}$ R. López-Ruiz, H. L. Mancini, and X. Calbet, Phys. Lett. A 209, 321 (1995).

${ }^{9}$ R. G. Catalán, J. Garay, and R. López-Ruiz, Phys. Rev. E 66, 011102 (2002).

${ }^{10}$ M. Perakh, On Talk Reason, www.talkreason.org /articles/complexity.pdf, August (2004); e-print arXiv:nlin/0701048v1 [nlin.CD].

11 J. Sañudo and R. López-Ruiz, Phys. Lett. A 372, 5283 (2008)

${ }^{12}$ J. Sañudo and R. López-Ruiz, J. Phys. A 41, 265303 (2008).

${ }^{13}$ C. E. Shannon, Bell. Sys. Tech. J. 27, 379 (1948); 27, 623 (1948).

${ }^{14}$ M. T. Martin, A. Plastino, and O. A. Rosso, Physica A 369, 439 (2006).

${ }^{15}$ J. Pipek and I. Varga, Phys. Rev. A 46, 3148 (1992).

${ }^{16}$ I. Varga and J. Pipek, Phys. Rev. E 68, 026202 (2003).

${ }^{17}$ A. Rényi, Proceedings of the 4th Berkeley Symposium on Mathematical Statistics and Probability, Vol. 1: Contributions to the Theory of Statistics, 1961, p. 547.

${ }^{18}$ C. Tsallis, J. Stat. Phys. 52, 479 (1988)

${ }^{19}$ S. Liu and R. G. Parr, Phys. Rev. A 55, 1792 (1997).

${ }^{20}$ S. Liu, A. Nagy, and R. G. Parr, Phys. Rev. A 59, 1131 (1999).

${ }^{21}$ E. Romera, R. López-Ruiz, J. Sañudo, and A. Nagy, Int. Rev. Phys. 3, 207 (2009).

${ }^{22}$ J. Pipek and I. Varga, Int. J. Quantum Chem. 64, 85 (1997).

${ }^{23}$ R. López-Ruiz, Biophys. Chem. 115, 215 (2005).

${ }^{24}$ L. Debnath and P. Mikusinski, Introduction to Hilbert Spaces with Applications (Elsevier, New York, 2005).

${ }_{25}^{25}$ A. Galindo and P. Pascual, Quantum Mechanics I (Springer, Berlin, 1991).

${ }^{26}$ H. A. Bethe and E. E. Salpeter, Quantum Mechanics of One- and Two-Electron Atoms (Springer, Berlin, 1977).

${ }^{27}$ C. Cohen-Tannoudji, B. Diu, and F. Laloe, Quantum Mechanics (Wiley, New York, 1977). 